Euskal ikerketen aldizkaria | Revue d'études basques |

Revista de estudios vascos | Basque studies review

19 | 2016

Numéro XIX

\title{
À la recherche de l'intestin aveugle : Ethno-texte sur la boucherie-charcuterie porcine en breton
}

\section{Nelly Blanchard}

\section{OpenEdition}

\section{Journals}

Édition électronique

URL : https://journals.openedition.org/lapurdum/3191

DOI : 10.4000/lapurdum.3191

ISSN : 1965-0655

Éditeur

IKER

Édition imprimée

Date de publication : 1 janvier 2016

Pagination : 103-119

ISBN : 978-2-95534-134-6

ISSN : $1273-3830$

Référence électronique

Nelly Blanchard, «À la recherche de l'intestin aveugle : Ethno-texte sur la boucherie-charcuterie porcine en breton », Lapurdum [En ligne], 19 | 2016, mis en ligne le 01 janvier 2021, consulté le 03 septembre 2021. URL : http://journals.openedition.org/lapurdum/3191 ; DOI : https://doi.org/10.4000/ lapurdum.3191

Creative Commons - Attribution - Pas d'Utilisation Commerciale - Pas de Modification 4.0 International - CC BY-NC-ND 4.0 


\section{À la recherche de}

l'intestin aveugle :

Ethno-texte sur la

boucherie-charcuterie

porcine en breton

Nelly BLANCHARD

Université de Brest / Centre de recherche bretonne et celtique

Drôle de manière de saluer la carrière d'un collègue que d'aller enregistrer l'abattage et la découpe d'un cochon. C'est que le dialectologue et lexicologue pointu du domaine basque, à qui nous rendons collectivement hommage par cet ouvrage, m'a un jour demandé si je connaissais la dénomination bretonne d'une partie bien précise de l'intestin dont le nom basque est motivé par « l'intestin aveugle ». Je ne pouvais lui apporter de réponse, ne sachant ni à quelle réalité physiologique correspondait cette partie de l'intestin, ni par conséquent comment elle se nommait en breton. Pour autant, il n'était pas question de rester dans l'ignorance et cet hommage est devenu l'occasion et le prétexte de me mettre à la recherche de ce mystérieux « intestin aveugle ». J'ai donc accompagné un boucher de campagne ambulant bretonnant, l'un des derniers à pratiquer ce métier de l'abattage et de la découpe du cochon dans les fermes, pour l'entendre me parler de son métier et collecter le vocabulaire de la boucherie et de la charcuterie en breton.

L'enregistrement a eu lieu en deux temps - les 4 et 24 avril 2015 - dans une même ferme d'une commune de Basse-Bretagne, aux côtés de ce boucher en retraite, doté d'une expérience de quarante-neuf ans de métier, et ayant toujours pratiqué l'abattage et la découpe de bêtes (cochons, vaches, veaux, moutons) à la ferme en parallèle de son activité de boucher en abattoirs ou en boucheries. C'est la méthode de terrain qui a été privilégiée, pensant que rien ne vaut la mise en pratique pour évoquer l'activité ciblée, reconnaître et nommer les parties du cochon, connaître les recettes de charcuterie, les outils, comparer les pratiques anciennes et actuelles autour de l'abattage et de la découpe du cochon. Les inconvénients liés à cette méthode sont la présence de bruits parasites, notamment ceux produits par certains outils qui couvrent parfois les paroles, la nécessité de concentration du boucher liée à la minutie de l'activité en cours et qui laisse parfois certaines phrases en suspend malgré une aisance du boucher à ne pas interrompre son travail lors de ses explications, et enfin la présence d'autres personnes (propriétaire bretonnant qui aide à l'abattage, acheteurs bretonnants et non-bretonnants faisant la mise en sac lors de la découpe et aidant à la préparation de la charcuterie) qui favorise la diglossie et une alternance codique selon les personnes auxquelles s'adresse le boucher ou la nature des conversations. À part les quelques adresses en français 
104

faites par le boucher au cochon lors de l'abattage, qui ont été conservées en italiques cidessous (car elles sont révélatrices d'un certain type de lien à l'animal), seules les paroles en breton du boucher ont été transcrites. Enfin, quelques brefs éléments de recontextualisation ou d'explication ont été fournis entre crochets ou en notes de bas de pages pour faciliter la compréhension du lecteur qui n'aura pas eu le plaisir d'assister aux scènes évoquées.

\section{Transcription en breton}

\subsection{Lazadeg ar penoc'h}

[E-barzh un hangar. Daou benoc'h a zo war ar plouz.]

Boñ, eomp da essae digass anezañ amañ ! Essae da gomañs! Hemañ eo hir e feskennoù. Troet eo mad. N'ouzon ket penaos e vo beteg ar fin, med... Kae da gerc'had ur sailhad ${ }^{1}$. Matrese e vo graet buannoc'h diouzhtu. Eh, t'es dans le mauvais sens, jeune homme! Hennezh a c'hallfe mond da bardon Sant Yann² : bale a ra!

[Kregiñ a ra ar c'higer en horzh.] Me, a skoan ${ }^{3}$ bremañ gant ar penn-mañ, gant an horzh. Ar bouetenn ${ }^{4}$ lem amañ - ar pezh a oa hirroc'h a-raog peogwir eo bet torret - a servije da lazañ al loened. Me, pa aen da lazañ aneze, a skoe ar fass gant ar penn karre, da ziskar anezañ. Ha goude gant hemañ e raen un toull e-barzh e dal. Da imobilisañ peogwir al loened gwechall a veze gwadet war an douar. Da imobilisañ al loen e passeen ur wazh rond e-barzh an toull da vont e liwenn ${ }^{5}$ e gein, da baralisañ al loen. Peogwir mod-all e talc'h ataw da voujañ hag e vezes taolet war da gein. [Skeiñ a ra meur a wech.] Kaled eo e benn! Ne vezan ket jentil ataw. Essae a ran da lakaad anezañ jentil, med... N'on ket tener gante.

[Trec'hiñ a ra e c'houzoug da wadañ ar penoc'h.] Mad eo, trawalc'h zo. Allez, remue maintenant! [Treiñ a ra ar gwad gant ur biz.] Abalamour dezañ pas koaguliñ. E 'h an da rekuperiñ les fibres. Goude, ar gwad a chomo likid. Lec'h ma n'em bije ket remuet anezañ e vije aet toud e memes tra pezh. 'H an da rekuperiñ petra... l'éponge a vez graet eus an dra-se. Ha bremañ ne goagulo ket ken. Bez a zo lod a-wechoù, abalamour deze pas remuiñ anezañ, a laka gwinegr e-barzh. Me n'am meus ket graet james.

Klewet ez peus anezañ o c'hwikal ? O, n'hon eus ${ }^{6}$ ket bet a boan. A-wechoù e vez muioc'h. A-wechoù e vez poan o tigass anezañ aze. Ar penoc'h a zo hinkin ${ }^{7}$, ar re-se a zo vis fall en o c'horf. Me am meus bet karget moc'h gwechall e-barzh ar gamionetenn, pa lazemp toud ar moc'h hom-unan, peogwir e 'h aemp da zastum aneze war ar maes, hag e vezent karget e-barzh ar gamionetenn. $\mathrm{O}$, ar gamionetenn a rae kazimant pewar-ugent sañtimetr uhelded, neuse

1. Ur sailhad boued da lakaad an eil penoc'h da c'hortoz trankil.

2. $/ \mathrm{s} \tilde{\varepsilon} \int \tilde{a} \mathrm{n} /$

3. Displegañ a ra ar c'higer alies ar verboù goude ar rener : hypercorrection ? Roudoù eus ur cheñchamant e-barzh ar yezh ? fazi ? Difaziet em eus goude e-barzh ar pennad skrivet.

4. Ar glaouetenn.

5. /livin i gejn/

6. /mœm/

7. N'eo ket sur eo ar ger-mañ peogwir e tistag /indi/ pe /indin/ (= int-i ?). 
e veze red dougen aneze, da lakaad aneze. Med a-wechoù e oa moc'h a seurt-se da zougen. Me a rae se gant ur plac'h, honnezh a oa deg vloaz ha tri ugent, etre hom daou o tougen ar penoc'h evel-se. E lakaen va dorn indan ar c'hof hag he a groge en e skouarn, hag e tougemp ar penoc'h evel-se, da lakaad anezañ e-barzh ar gamionetenn, peogwir ne oa pontenn ebet, e oa kont dibradañ aneze. Med ur wech o zreid war ar c'haled, alies e 'h aent, e oa aeset d'ober. Peogwir ar gamionetenn a oa uhelloc'h ewid an oto aze. Ma tegoueze dit lakaad penn e benn e-barzh, un troad a-raog e-barzh, ur pezh taol dorn war e gein, e rae ur pezh lamm e-barzh. Ha bremañ n'eo ket aeset dougen aneze abalamour d'o lostoù : al lostoù zo berr. Abalamour ma vez troc'het o lostoù deze, peogwir e vezont savet e-barzh des calibotis, ma valeont an eil war lost egile e wadont, ha ma stagont warne, ar re all a 'h a ataw da sugellad o gwad deze.

[Dour a zo bet lakaet da verwiñ gant ar proprietour.] O verwiñ ataw e oa? 'H an da skaotañ e dreid. Komañs a ran ataw da skaotañ o zreid deze da zigentañ. An tammoù diaessañ da zigentañ. Pa vez braw an dour, $90^{\circ}$ normalamant, eo trawalc'h da skoatañ ar penoc'h. Ma vez re domm re domm e poaz ha neuse ne deu ket mad. Normalamant ar yer a vez graet gant 75-80'. Ma teues da boazañ aneze e teu ar c'hroc'hen ganit.

[Direunañ a ra ar penoc'h.] Ar vicher-mañ eo direunañ ar penoc'h. An ordr a zo ataw heñvel. Pa n'en devez ket a ordr ne res seurt ebet. An traoù diaessañ ataw da gentañ. An treid, an diwskouarn hag ar penn. Ha goude ar c'horf. Gant ar gontel espres e vez graet. Un peleux a vez graet eus an dra-se. Med houmañ am meus bet torret, houmañ zo bet ur gontel da drec'hiñ kaol hag em meus ingalet anezi d'ober... N'eo ket a boan anezi trec'hiñ. Ar reun, sañset, a vez tennet. Ar gontel-se ne droc'h ket dutoud.

An dra-mañ a zo eveltomp iwe : bez a zo le derme ha l'épiderme. Ni zo evel-se iwe. Ar c'hroc'hen kentañ, normalamant, a deu kuit da heul ar reun. N'en devez ket riou oc'h ober ar vicher! N'eo ket a boan gortoz anezañ da yenañ. Ewid ar c'hroc'hen bihan surtoud. Ma vez yenet ar penoc'h, se zo evel pluñvañ yer iwe: ma lazan kalz re a yer assambles e ran aneze deg ha deg peogwir n'eo ket a boan yenañ aneze, peotramant goude pa lakaes aneze en dour tomm, an diabarzh a gomañs yenañ, hag o tikroc'henañ aneze e teu toud ar c'hroc'hen ganit. Hag amañ heñvel iwe, toud. Ne dalvfe ket a boan direunañ ar penoc'h an deiz war lerc'h. Ar pezh ne deu ket, ar chalumo a okupo deus oute damaig. Evel pa basses ar poule war an tan.

Impeccable! N'eo ket divalo! Hemañ a zo troet, paotr! Flamm eo. En bew, hennezh a ra kant ha pewar-ugent lur ${ }^{8}$. En kig e rafe apeurpre evel poues ar re all iwe : kant tregont lur, da neubeutañ. E ro kig, ar paotr!

Da lakaad anezañ a-istribilh e tapan an nervennoù, a-dreg e dreid : an tañdoñ. Deus un tu e vez lakaet ar gordenn peogwir e 'h a deus forc'h an trakteur, ha mod all gant ar palañ a beb tu, peotramant, mod all, e lakan anezañ war ar pantouar. Gant an trakteur eo aeset. Aeset da bourmen anezañ. [Lakaad a ra dour warnañ.] Red eo delc'her anezañ naet. 'H an da basseal un taol chalumo warnañ da dapout ar ful. N'eo ket a boan iwe da vont gant ar chalumo lec'h m'eo lous, peotramant e chomo lous.

8. Ul lur a dalv kement hag un hanter kilo (pe un tammig nebeutoc'h), med ewid ar c'higer-mañ e talv kement hag ur c'hilo, war a seblant. 
Poent eo faoutañ ar penoc'h. Bremañ e vo digoret. Forzh penaos, digoriñ ar penoc'h e vez ataw heñvel. Aze forzh penaos e tigores an diwvorzhed. Ha goude e vo digoret penn-hapenn : an operasion vras! Moarvat, deomp-ni e vez graet heñvel pa vezomp operet iwe. Kar pa vezomp operet e vez lakaet toud hom bouelloù war an daol tal-kichen.

Ar bouelloù a vez chetet war ar bern teil pe lakaet en douar. Hennezh eo ar vouellenn dall. Ar penn e 'h on da okupiñ deus outañ, e 'h an da droc'hiñ e benn dezañ. Amañ emañ an empenn. Deja e vigorn toud. Tennañ a ran foñs an diwskouarn. An abatoarioù ne reont ket, med gant ar serumen e kavan gwelloc'h. Ar penn da zistrempañ. Ar gwad er frijider. Un tamm skubellenn. Propre pour le prochain! N'omp ket bet pell. Se a zepend deus ma tro an traoù.

[Droed az peus d'ober ar vicher-se ?] Droed zo war ar maes, med normalamant zo droed med ewite o-unan pe d'o amezeg. N'eus ket droed d'ober koñvers gante. Med ar re-se zo droed, n'int ket bouklet na seurt ebet. Ar re-se zo deklaret toud consommation familiale.

[Trubuilhoù az peus bet ?] Me n'am meus ket bet james. Re all, kalz re all o deus bet. Me am meus bet muioc'h a chañs ewid ar re all : n'am meus ket bet. Me ne ran ket forzh. An hini n'eo ket kontant n'en deus ket med dont da rañplasiñ ahanon. Me a zo kontant da laoskel un all d'ober, da zeskiñ d'un all ober al labour. [Kavoud a res tud da zeskiñ ?] O, poan a vo kaoud. Unan a lâre din e-barzh ur ferm dec'h : «Pa n'aefi ket ken, penaos 'vo graet ? » Ur gwall vicher eo hag honnezh a areto evel ar re all iwe. Red eo goûd lazañ aneze. N'eo ket a boan kaoud aon, kwa. Me am meus komañset ar vicher-mañ... ar mis-mañ e ra naw bloaz ha daou-ugent on oc'h ober ar vicher-mañ.

[Soñj az peus eus ar wech kentañ ?] O ya ! Evel ma vije bet dec'h! Red e oa kaoud tamm karakter, bezañ solud da gentañ. Med boñ, me a oa diwar ar maes. Ha koulskoude, pa veze lazet ar penoc'h er gêr gwechall, ne chomen ket da lazañ ar penoc'h, me a 'h ae kuit. Med abaoe... An dra-se zo evel un infermierez pe ar medesin : ar re-se a vez o trailhañ tud. Heñvel. An dra-se am befe poan o suportiñ, gweled a res. Me n'am meus ket med ur penoc'h etre va daouarn. Ar re a vez buhez un den etre o daouarn eo gwassoc'h. Med boñ unan bennag a rank ober anezañ. Bezañ labouret en abatoarioù n'eo ket labour deus ar re vrawañ ataw iwe. Pa vez gwelet ar marc'hadour evel-se, kaoud ar gigerez pe ar chakutiri, pe traoù evel-se, an dra-se a vez mad, med ne vez ket gwelet petra labour a vez a-raog.

\subsection{Dispennadeg ar penoc'h}

E 'h eomp d'ober gwenediged'. Gwenediged a vez graet gant gwad, kig sall druz hag ognon. Ewid pewar lur a wad penoc'h : pewar lur ognon, tri lur kig druz, ha goude apeurpre tri litrad gwad, pewar litrad gwad. Red eo laoskel aneze da boazañ da gentañ, da rostañ, da fritañ un tamm bihan.

Pa vez komañset dispenn ar penoc'h e vez ataw memes tra reus : e vez troc'het pewar pe bemp tamm da gentañ, d'ober pezhioù, a-raog dispenn an tammoù bihan. " Disober » am meus bet klewet iwe, med me a ra « dispenn » deus an dra-se. Me am meus klewet ataw « dispenn ar penoc'h».

9. Mod ar vro da lâred « gwadigennoù ». 
E 'h an da dennañ ar file kuit. Gwechall, pa veze dispennet ar penoc'h, e veze an tamm da vezañ debret ataw deiz an dispennadeg. Hennezh a oa ar fest ataw gwechall, an tradision. Ar geginerez a lakae ataw hennezh da brepariñ al lein. Ha forzh pelec'h e oa ar c'higer, gwechall e oa evel-se. An tradision a oa debriñ hennezh assambles gant ar c'higer. Med bremañ an traoù zo cheñchet. An dispennadeg n'eo ket heñvel ken. Gwechall pa veze dispennet ar penoc'h e veze lakaet toud ar c'hig - ne oa ket koñjelateurioù - toud ar c'hig e-barzh ar pod, Hag e veze distaget ar c'hig treut deus ar c'hig druz, peogwir ar c'hig druz a veze lakaet e foñs ar pod. Mod all e veze graet kostiennoù kig-sall, e veze lakaet an dra-se a-istribilh. Med ar moc'h a veze kalz ponnerroc'h gwechall ewid bremañ, kalz druoc'h, peogwir seul vui ma veze druz, e veze lâred « an ti a oa pinvidig ». Pa veze gwelet ar c'hig-sall a-istribilh, opala, « hemañ zo un ti mad».

Hennezh eo ar bruched. Ar c'hostiennoù kig-sall, kwa. Distagañ anezañ deus ar skoaz. Ne veze ket graet kostiennoù evel bremañ, e veze graet mell-kein ${ }^{10}$. Mell-kein a gorespond e veze troc'het ar c'hostiennoù a-rez da liwennoù ar c'hein, da liwenn-gein al loen, deus an daou du e veze laosket da vont an druz kreiz ar c'hein. Hag e veze lakaet ataw war c'horre ar pod, abalamour da zebriñ aneze da gentañ. Alies, an tradision da ingalañ gant an amzeien a oa reiñ un tammig kein d'an amezeg ataw pe d'ar familh, traoù evel-se, d'ober plijadur deze. Kar hennezh a veze an tamm gwellañ. Mez goude e veze alies, pa veze d'an amezeien da zispenn iwe, ar gompensasion a veze restorniñ anezañ. E gis-se oa an okasion da zebriñ kig fresk e-pad pelloc'h. Peogwir ne oa ket sort da gonserviñ anezañ.

Peb kontell, normalamant, he deus he labour. Bez a zo lod eus ar c'hontelloù a zo graet da ziaskornañ, lod all a zo da beilhad ar c'hig, ha lod all a zo da trañchiñ anezañ d'ober tammoù bras. Hag an heskenn da drec'hiñ an askorn. Hag ar feuilhenn da droc'hañ ar c'hostiennoù. Ha da faoutañ ar penoc'h. Ar feuilhenn a zo un hach bihan. Mod all, ewid al loened-korn eo ar c'houpre, kalz brassoc'h. Lemañ ar c'hontelloù, an dra-mañ a zo ur vicher all iwe. Red eo goûd ober. Ma ne lemes ket mad, an dra-se eo diwar da goust goude. An dra-se a zegouez a-wechoù bezañ stoket. A ra parti eus ar vicher. An taol gwassañ am meus bet eo un taol gontell em gar. O wadañ ar penoc'h, evel em meus graet aze. Hag ar penoc'h a oa degouezet dezañ mont...Ebarzh an abatoarioù e vez toulloù da rekuperiñ ar gwad, un toull e-barzh ar simañt, a peupre da lakaad ur sailh e-barzh un toull rond evel-se. Neuse e lakaes ur sailh e-barzh da rekuprin ar gwad. Ar penoc'h a oa degouezet dezañ mont e-barzh evel-se ha me o kregiñ e-barzh troad ar penoc'h evel hemañ. Ar gontel a oa em dorn. Hag ar penoc'h ne oa ket marw c'hoazh. Ha pa am meus sachet anezañ er maes, gant ur gontel evel houmañ, ar penoc'h en doa graet ur sach-krenn warnon evel-se ha pof, riklet ar gontell. Aze e oan bet seiz mis gant an dra-se. Med ne oan ket bet aretet. Bremañ zo kalz a aret a-raog an dra-se.

Pa vezomp deus ar vicher - ar re a zo deus ar vicher - e anavezomp ur penoc'h mad, ur penoc'h druz. Deus ar stumm. Gwechall pa brenemp loened war ar maes, peogwir e veze prenet loened war ar maes, e vezent toud prenet a-bak, e vezent prenet gant ar c'hlassamant. Ne oa ket ezomm da anavezoud kalz peogwir goude e vezent kasset d'an abatoarioù. Med ni, gwechall, pa brenemp aneze, a brene aneze gant hom daoulagad. E veze prenet priz a-dreuz.

10. /milkzjn/ : ewid ar c'higer e talv kement ha kreiz (mil-) ar c'hein. 
Ha deomp da c'hoûd pe oa un mad pe ne oa ket. Ha petra poues a oa apeupre. Me am meus soñj : deus stumm al loened gwechall, war re neubeud, o ya! war deg lur apeupre, e ouien petra poues kig e oa al loen pa veze lazet. Gwechall, pa oa al loened-korn, pa veze ar marc'hadour da brenañ aneze, a c'houlenne an aliessañ d'ar proprietour sortial al loened deus o c'hraou da c'hoûd petra poues a raent. Peogwir an taol-lagad n'eo ket heñvel seloñ ar c'hraou. Pa vez ur c'hraou gant un doenn uhel e kav al loen kalz bihannoc'h, hag e-barzh ur c'hraou bihan e kav anezañ kalz ponnerroc'h. Stumm ur penoc'h mad? A vez gwelet mad eus e feskennoù, troet mad, ha deus liwenn e gein. Surtoud liwenn e gein. Normalamant a-benn kaoud ur penoc'h mad eo daw kaoud ur penoc'h hir. Hag ewid ur penoc'h ekstra e vo faoutet kreiz e gein kazimant, e vo ur wazh e kreiz e gein. Neuse e vo unan passe ekstra. Se zo kaos, an taol-lagad a gont kalz. [Liw ar c'hig ?] O ya, goude, ya. Med boñ, ar c'hig, nemed e vefe un hoc'h gae ${ }^{11}$ - un hoc'h gae a zo un penoc'h na vez ket troc'het, bremañ ar moc'h a vez troc'het -, mod all e vije kig kalz druoc'h. Mod all ar c'hig-penoc'h a zo kig gwenn kazimant.

[Loened diform, a-dreuz ?] An dra-mañ a zo en em gavet ganin alies : kostiennoù torret alies. Alies pa vezent bihan, bet baleet o mamm warne. Hag e vezont diform un tamm bihan. Med gwelloc'h eo, en em zougen e-unan. Alies int bet evel-se gwasket en bihan. Mod all a-wechoù e vez kleñvedoù. Gwechall e veze kaos eus an tuberkuloz un tamm bihan. Me am meus soñj pa oamp o labourad, pa lazemp loened, pa veze speg ar poumonoù deus ar c'hostennoù, hag en abatoarioù e vez ataw evel-se : an dra-mañ a vez « suspect ». Mod all e ouiemp koulz hag ar vetrinaer pe e vije mad ar c'hig pe ne vije ket. Bez hon neus gwelet un tamm a bep seurt e-barzh hom micher. Proprosionet eo al loened apeurpre, war re neubeud. Par contre, ar rognonoù, an dra-se am meus bet gwelet alies - e vez gante evel gant an dud - ne veze ket med unan, petramant e veze unan disec'het. Matrese en bihan int bet ganet evel-se. Hag egile un hanter brassoc'h. Moarvat peogwir en deus an hanter muioc'h a labour. Hag an dra-mañ a droc'homp ouzhpenn d'ar penoc'h d'ober ar charkutiri, ar silzig gante, peotramant anduilh, anduilh-kig. Anduilh a vez graet nebeutoc'h peogwir n'eus ket chiminaloù ken da vogediñ aneze.

[Loened distumm ?] Ket. An dra-mañ n'am meus ket bet gwelet. A-benn ma weler un dra a seurt-se, alies ar re-se ne vewont ket kozh, matreze e tegouez deze merwel a-raog. Aksidantet em meus graet. Gwechall, peogwir e oa droed da lazañ war ar maes, beb taol, surtout d'ar poent-se deus ar bloaz, e veze leun d'ober. Pa veze kasset an onnered er-maes, neuse e aent e-barzh an hentoù don ha torret o diwhar. Peogwir al loened a veze savet gwechall e-barzh kreier du, peogwir ne oa prenestr ebet. Peogwir gwechall e veze red stouvañ ar prenestroù abalamour d'al loened da gaoud tomm. Bremañ eo ar c'hontrol, bremañ eo red aweliñ. Neuse pa gasses aneze er-maes, ne welent ket sklaer. Neuse poent ar bloaz-mañ e veze alies ur bern saout ... Pa veze ar saout oc'h alañ e raent des hémorragies internes. Ped gwech n'on ket bet e noz goulennet da lazañ ur vuoc'h. N'on ket bet beb noz em gwele. Pa lazen aneze e tigroc'henen aneze, ne oant ket falloc'h da zebriñ, un hémorragie a raent med ne oant ket falloc'h da zebriñ. Bremañ loened a seurt-se n'eus ket droed ken da lazañ war ar maes, an écarissage o tont. Med an dud gwechall..., peogwir ar vetrinaer a veze o hopal warnon,

11. /je:/ 
peogwir eñ pa en em gave, alies e vez goulennet ar vetrinaer da vont da weled al loen, ma vez seurt ebet d'ober, eñ an hini eo a sutele warnomp, evel-se e soulaje proprietour al loen peogwir an dud gwechall ne oant ket pinvidig, koll ul loen a oa ker deze. An traoù a zo cheñchet. Hemañ a zo ur gwall vicher. Ha n'eus ket kalz a goñkurañs. N'eus den ebet ken.

An tamm druz a zo war liwenn e gein a servij d'ober ar paste. Toud ar c'hig druz a vez lakaet alies e-barzh paste. E tammoù an druz toud peogwir, pa vez graet ar paste kañpagn, e vez graet apeupre daou lur kig druz ewid ul lur foilh hag ar galon.

Gwechall pa veze lazet ar penoc'h ne veze tamm koll ebet, peogwir memes - me am meus soñj gweled va zud oc'h ober peogwir ar c'higer a deue, hag ur c'higer a oa e kement kartier zo toud - a rae nemed dispenn ar penoc'h hag ober ar podad kig. Ha goude e veze da bebhini ober e charkutiri e-unan. Me am meus gwelet boulboazet pe hanter boazet an donenn lakaet e-barzh ar paste. Bremañ e tennomp anezi kuit : an dud zo difisil ! Lard teuz ${ }^{12}$ ne vez ket graet kalz ken. Me am meus graet n'eus ket keid-se a zo, peogwir em boa kavet unan vraw. Evel-se da lakaad war ar bilig d'ober krampouezh. Peotramant pa vez graet patates newez e-barzh ar chidourenn. Ar vroneg ${ }^{13}$ eo pa lakaes anezañ a-istribilh. Pa veze lakaet an traoù a-istribilh gwechall, e veze graet « broneg » eus an dra-se. Paket en ur file ha lakaet da zisec'hañ, hag e veze tapet, troc'het tammoù diwarnañ. Ha lard dous eo ar c'hig sall n'eo ket sallet.

Ar charital : sall un tamm bihan, neuse vezomp ket feneanted ouzh taol ! Bez a zo broioù a ra jamboñioù sec'h med an dra-mañ n'eus ket a voaien d'ober amañ. An amzer a zo re humid. Hag e vreinont, ne sec'hont ket. Rotioù, roti-penoc'h. Kostiennoù e 'h an d'ober iwe. Hag a-wechoù, an tamm-mañ a vez graet ar choukenn deus outañ, goug ar penoc'h. Tennañ ar c'hostennoù d'ober treñchennoù goude.

Bremañ e 'h an d'ober ar paste kañpagn. [Ober a ra tro an tammoù a zo en ur vasin.] Amañ emañ an empenn. Hag unan en deus c'hoant da gaoud an empenn ? Den ? Intelijañt awalc'h oc'h ? Amañ emañ ar rouedenn, a vez lakaet war ar paste. Houmañ a vez stag ouzh an estomok. Da lakaad war gorre ar paste goude. «An tor» a vez graet eus an estomok. Ar galon. Ar poumonoù. Gwechall e vezent lakaet e-barzh ar paste iwe. Gwechall ne veze kollet seurt ebet. «Ar skevent» a gle bezañ ar poumonoù. An evu. [An evu e-barzh ar paste ?] Gant petra e ri paste neuse? Ma n'az peus ket foilh, n'az peus ket paste. An dra-se eo paste evu. Pastepenn ha paste-penoc'h, peotramant paste-penn all iwe. Ar paste a zo prest. Bremañ n'eus ket med drailhañ anezañ d'ober.

[Draillerezh-dorn.] An dra-mañ am meus graet, e komansamant va micher, gant an dra-se. An dra-se a oa ur vicher difonn! Hag e oa red kaoud un tamm nerzh. Ha kaoud un daol solud peogwir e oa red stagañ ar machin ouzh an daol. Gwelet am meus ur wech, un ur plass, e oa stag un daol ouzh un espes cloison, ha peb tro deus ar manivel e tistage un tamm eus an daol : brein, kwa! Med e-barzh toud ar menajoù a zo ataw un draillerezh evel-se, kazimant : un draillerezh-dorn e-barzh toud ar menajoù.

[Ar bouelloù.] Gwechall ha bremañ memes, eo gant ar bouelloù moan e vez graet ar

12. /lart $\tilde{\varepsilon} /$

13. /ar vrunik/ 
silzig. Ha gwechall e veze graet gant ar bouelloù bras, boueloù tew, e veze graet an anduilh gant kig da lakaad a-istrilh. Med bremañ ne chomer ket ken da walc'henniñ se. Eo, bez em meus c'hoazh daou a ra ataw diw anduilhenn-bouelloù gante. Med boñ, ne ran ket kalz. Bremañ an anduilh bras a vez graet gant bouelloù loened-korn, hag ar bouelloù silzig gant bouelloù moc'h. Bremaig, da weled iwe, e 'h an d'ober silzig moan, chipolata, ar re-se a zo graet gant bouelloù deñved. Ar c'hig a zo heñvel, med ar bouelloù n'int ket heñvel. Med bremañ ar re yaouank a 'h a gwelloc'h gante ar silzig moan. Koulskoude ar re dew o deus kalz gwelloc'h blaz. [Gwalc'hiñ ar bouelloù ?] Ne glaskan ket ober peogwir e c'houl re a amzer. Se zo kaos bremañ ar bouelloù a goust ker hag e vez graet industrielamant. Me a walc'he aneze gant an dorn gwechall.

[Ar bouelloù.] Ar bouelloù bras a zo, med goude ez peus ur vouellenn evel ur sac'h e penn, evel ur « poch » a veze graet deus an dra-se : « la poche d'appendice ». Gwechall n'ouzon ket hag-eñ ne veze ket graet ar vroneg e-barzh. Ar vroneg a veze graet gant ar saindoux, a veze paket hag a veze lakaet da vogediñ er chiminal, hag e veze laosket a-istribilh evel-se. A veze graet « ar vroneg » eus an dra-se. An dud, gwechall, a droc'he tammoù da lakaad e-barzh ar soubenn. Bremañ moarvat e vije poan o kaoud tud ewid seurt-se. Ar vouellenn dall, hounnezh zo ur vouellen dall, ya. A c'heid-se apeupre [diskouez a ra un ugent sañtimetr bennag gant e zorn]. Ur pochad a ra hag ar boued a 'h a e-barzh, er sac'h golo, kwa. Ha goude e rank dont war e c'hiz da sortial dre an toull-se. Sed, ni a zo evel-se iwe. N'on ket bet o weled! Ha ne garjen ket gweled, memes!

[Poaz eo ar penn.] Tomm an traoù ! Ne welan ket sklaer iwe e-barzh. Tro deg eur ! [Un ehan, ur banne gwin, da c'hortoz ken ma vo yennaet un tamm ar penn.] Kemeret un tamm nerzh, bremañ eomp adarre. [N'az peus ket naon ?] O nann, me a zebr re vad deus ar beure. Mod all, traoù evel-se ne ro ket a galon eus a debriñ.

[Paste kañpagn.] Ale, an holen hag ar pebr. An dra-se zo evel pa sales ar soubenn, e res apeupre iwe. Ne lipan ket. Ar paste-penn damaig.

[Petra a zo mad da zebriñ er penn ?] Toud med an askorn. Ar chotennoù, ar fri hag an teod. Ket an daoulagad, mod all e chomo ar penoc'h da selled diouit ataw. Amañ al lagad, pa vez poaz mad n'eo ket diaes da ziaskorniñ. Ur fesson a zo da gemer aneze, ur señs iwe. Dent an hini eo a vank dezañ ! Ar machin ne gar ket kalz an askorn. Ar bigorn e-barzh an diwskouarn.

[Gwadigennoù.] Piw en deus c'hoant teñvañ anezañ ? N'eo ket med gwin tew ! N'eo ket fall. Ma vez tomm mad awalc'h an dour, deg munut, ur c'hard eur da boazañ. Ma vez tomm tomm an dour. Me a boaz anezañ e-barzh ar bouilhoñ a zo bet da boazañ ar penn. Hennezh eo red surveilhiñ anezañ un tamm bihan. N'eo ket a-boan komañs berwiñ peotramant e vo sin fall dezañ. Mod all e eklatont toud, ne chomo ket unan en e bezh.

[Penaos ez peus desket ar vicher ?] Me am meus desket va micher war ar bern, en apprentissage ha des cours par correspondance, la technologie a oa toud par correspondance. Me a oa e skol Gambetta M., un dewezh bep pemzektez: O ! ne oa ket re! D'ar poent-se e veze graet an apprentissage ewid tri bloaz hag e penn an daou vloaz e oan aet da dremen ma CAP kiger, ha peogwir oa tri bloaz apprentissage, ar bloaz goude em meus graet ar chakutiri. Al live skol ne oa ket deus ar re uhellañ. Bremañ, ar re a deu er-maes eus ar skol, ewid ar CFA e B., bremañ zo kalz muioc'h a skol ewid ne vezont war ar vicher. Ni, goude tri bloaz, e oamp apt d'ober forzh petra. Lec'h, ar re a deu eus ar skolajoù, o deus ar vicher da zeskiñ c'hoazh. Desket e vez traoù deze er skol, med etre ar skol ha war ar vicher goude, war an dachenn n'eo ket heñvel.

Ar saout, an dra-mañ am meus graet kalz. Keseg am meus bet lazet iwe. Lazañ keseg 
am meus bet graet ewid an asurañsoù. Peogwir gwechall ar c'heseg, war ar maes, a veze asuret, hag e veze des groupements, toud an amezeien, ar sekteur amañ a rae parti eus X. Pa varve ul loen-keseg, matrese e oa hanter kant proprietour keseg, ha pa vanke ul loen-keseg en un tu bennaket, e veze evaluet al loen-keseg ur wech ar bloaz. Da lun fask e veze ataw an dra-se. Er rassemblement, en ur c'hroaz-hent, e veze estimet al loen-keseg. Setu evel-se, ma varve al loen-keseg war ar bloaz... Ha goude, pebhini a rañbourse al loen-keseg e proporsion e nombr a geseg en devoa. M'en devoa tri, war matrese kant loen-keseg, e vije diviset. Pebhini a roe un tamm gwerz-butun. Un tamm arc'hant, da rañplasiñ al loen-keseg. Peogwir ar c'heseg o devoa un dalvoudegezh gwechall. Goude zo bet an asurañs, evel an asurañsoù hon eus bremañ. Med boñ, bremañ n'eus ket loen-keseg ebet ken neblec'h. Keseg am meus bet lazet peogwir, d'ar poent-se e veze graet «chair de poisson » deus an dra-se. An dra-se a deu de la paralysie. An drase a veze gwelet alies e-barzh al loened-korn, se am meus bet gwelet pa oant bet aksidantet. Ar muskloù pa vezont atrofie petramant bet freget, a ra evel ur voul gres. E-barzh al loen-keseg e veze gwelet an dra-se pa vezent uset peogwir ar c'heseg a laboure kalz gwechall, hag e veze graet kig-pesked peogwir ar c'hig a veze gwenn. Hag a-benn bezañ rañbourset gant an asurañs e oa red deze bezañ marw. Paotred an asurañsoù a deue da glask ahanon da lazañ aneze. Me ne vezen ket paeet, e raen an dra-se bénévolement.

[Oc'h ober silzig.] Ar pousoar : ur wask. Ne vo ket lakaet toud e-barzh, red e vo ober diw dro. Ar re-se eo ar bouelloù moc'h. Amañ em meus bouelloù loened-korn d'ober an anduilh. Ar re-mañ a zo kalz tewoc'h, an diametr a zo kalz brassoc'h. Ale, mil vad. Penaos eo kont gant ar silizig ? An dra-se am meus graet : tournailhoù, des tonnes. Pa ouean petra poues am meus e ouean apeupre ped silzigenn e 'h an d'ober a-raog komañs. Normalamant gant ar pezh em meus aze e glefen ober apeupre kant ha daou ugent. Ar moc'h ne vez ket cheñchet. Me a lâr se ewid toud an traoù : an dud zo cheñchet med an natur n'eo ket cheñchet.

\section{Traduction en français}

\subsection{Labattage du cochon}

[Dans un hangar. Deux cochons se trouvent sur la paille dans un enclos.]

Bon, on va essayer de l'amener ici ! Essayer, pour commencer ! Celui-ci à les fesses allongées. Il est bien formé. Je ne sais pas ce qu'il vaudra au final, mais...Va lui chercher un seau de nourriture ${ }^{14}$. Peut-être qu'on y arrivera plus vite comme ça. Eh, t'es dans le mauvais sens, jeune homme! Il pourrait aller au pardon de Saint-Jean ${ }^{15}$ : il marche comme au défilé.

[Le boucher saisit une masse.] Maintenant, je frappe avec ce bout-là de la masse. L'embout pointu - le morceau était plus long auparavant, mais il a été cassé - servait à tuer les bêtes. Moi, quand je m'apprêtais à tuer le cochon, je frappais la face avec le bout carré, pour le mettre à terre. Et ensuite, avec cet embout, je lui faisais un trou dans le front. Pour

14. Pour que le deuxième cochon reste tranquille le temps de faire sortir le premier cochon de l'enclos.

15. Le pardon de Saint-Jean-du-Doigt (Finistère) a lieu le dernier dimanche de juin. 
l'immobiliser, parce qu'autrefois on saignait les bêtes par terre. Pour immobiliser l'animal, je passais un roseau dans le trou pour atteindre sa colonne vertébrale, pour paralyser l'animal. Car sinon, il continuait de bouger et il te renversait. [Il frappe plusieurs fois sur la tête.] Il a la tête dure! Je ne suis pas toujours gentil envers eux. J'essaie de les calmer, mais... Je ne suis pas toujours tendre envers eux.

[Il lui coupe une partie du cou pour le saigner et récupérer le sang.] C'est bon, il y en a suffisamment. Allez, remue maintenant! [Avec un doigt, il tourne le sang récupéré dans une bassine.] Pour qu'il ne coagule pas. Je vais récupérer les fibres ${ }^{16}$. Le sang restera ainsi liquide. Alors que, si je ne l'avais pas remué, il aurait formé un bloc. Je vais récupérer... comment déjà ? On appelle cela l'éponge. Et maintenant il ne coagulera plus. Parfois, certaines personnes, pour ne pas à avoir à remuer le sang, versent du vinaigre. Moi, je n'ai jamais fait ça.

Tu l'as entendu piauler? Oh, ça n'a pas été difficile. Ça l'est parfois davantage. Parfois, ce n'est pas facile de l'amener jusque-là. Le cochon, c'est coquin, ils ont du vice dans le corps. Autrefois, j'ai chargé des cochons dans une camionette quand on tuait tous les cochons nousmêmes, parce qu'on allait les ramasser à la campagne et on les chargeait dans la camionette. Oh, la camionette faisait environ quatre-vingts centimètres de haut, il fallait donc les soulever pour les y mettre. Mais parfois il y avait des cochons de cette taille-là à soulever. Je faisais ça avec une femme qui avait soixante-dix ans, et à nous deux on portait le cochon comme ça : je passais la main sous son ventre et elle, elle accrochait une oreille, et on portait le cochon ainsi pour le mettre dans la camionette. Comme il n'y avait pas de passerelle, il fallait le soulever. Mais une fois mis les pattes sur le dur, ils y allaient, c'était facile à faire. La camionette était plus haute que cette voiture. Si tu arrivais à lui mettre l'avant-train dedans, un pied dedans, une grande tape sur le dos, et il rentrait dedans en faisant un grand bond. Aujourd'hui, ce n'est pas facile de les porter à cause de leurs queues : ils ont les queues courtes. On leur coupe la queue parce qu'ils sont élevés sur des calibotis, et si les uns marchent sur la queue des autres, ils saignent et s'ils se mordent, les autres n'arrêtent pas de leur sucer le sang.

[Le propriétaire a mis de l'eau à bouillir tôt le matin.] Elle bouillait encore ? Je vais lui ébouillanter les pieds. Je commence toujours par leur ébouillanter les pieds en premier. Les parties les plus difficiles en premier. Si l'eau est bouillante $-90^{\circ}$ normalement - c'est suffisant pour ébouillanter le cochon. Si l'eau est vraiment trop chaude, alors ça ne vient pas bien. Normalement, on fait les poules à $75-80^{\circ}$. S'il t'arrive de les cuire, alors tu arraches la peau.

[Il rase la soie du cochon.] Cette activité, c'est raser le cochon. Lordre pour le faire est toujours identique. Quand on n'a pas d'ordre, on ne parvient à rien. Les choses les plus difficiles toujours en premier : les pieds, les oreilles et la tête. Et ensuite le corps. On fait ça avec un couteau spécial : on appelle ça un peleux. Mais ce couteau-ci, je l'ai cassé, c'était un couteau pour couper les choux et je l'ai égalisé pour le faire... Il n'est pas nécessaire qu'il coupe. La soie, normalament, on la tire. Ce couteau ne coupe donc pas du tout.

C'est comme pour nous : il y a le derme et l'épiderme. Nous, on est comme ça aussi. Lépiderme $^{17}$, normalement, vient avec la soie. Ça donne chaud, ce métier! Il ne faut pas que

16. Les passages en français du discours en breton sont indiqués en italiques.

17. Mot-à-mot : la première peau. 
l'animal refroidisse. Surtout pour l'épiderme ${ }^{18}$. Si le cochon refroidit, c'est comme quand on plume les poulets : si je dois tuer vraiment beaucoup de poulets en même temps, je les fais dix par dix parce qu'ils ne doivent pas refroidir, sinon ensuite, quand tu les ébouillantes, l'intérieur commence à refroidir et, quand tu les plumes, tu arraches toute la peau. Et dans le cas du cochon, c'est tout à fait pareil. Il ne faut pas attendre le lendemain pour raser le cochon. Ce qui ne vient pas [au couteau], c'est le chalumeau qui s'en occupera tout à l'heure. C'est comme passer la flamme sur le poulet.

Impeccable! Il est pas mal ! Il est bien foutu, le gars. Il est bien blanc. En poids vif il fait cent quatre-vingts kilos. En viande, ça ferait à peu près le poids des autres ${ }^{19}$ : cent trente kilos au moins. Il va donner de la viande, le gars!

Pour le suspendre, je saisis les nerfs à l'arrière des pieds : les tendons. D'un côté, on glisse une corde parce qu'il sera suspendu à la fourche du tracteur ; sinon, je l'attache au palan des deux côtés, ou encore je le mets au pendoir. Avec le tracteur, c'est facile. Facile de le trimbaler. [Il nettoie le cochon pelé à l'eau.] Il faut qu'il reste propre. Je vais lui passer un coup de chalumeau pour retirer le duvet. Et il ne faut surtout pas passer le chalumeau sur des endroits sales, sinon, ça restera sale.

Il est temps de fendre le cochon en deux. Maintenant on va l'ouvrir. De toute façon, ouvrir un cochon consiste toujours en la même chose. Là, il faut ouvrir les cuissots. Et ensuite on l'ouvre d'un bout à l'autre : la grande opération! Sans doute qu'on nous fait la même chose à nous aussi quand on nous opère. Parce que quand on nous opère, on nous met toutes les tripes sur la table, à côté !

[Il récupère les boyaux.] On jette les boyaux sur le tas de fumier ou on les enterre. C'est celui-là le cœecum ${ }^{20}$. La tête, je vais m'en occuper, je vais lui couper la tête. La cervelle est là. Elle se plisse déjà. Je retire le fond des oreilles. Les abattoirs ne font pas ça, mais à cause du sérumen, je trouve mieux de le faire. La tête à tremper. Le sang au refrigérateur. Un coup de balai. Propre pour le prochain! On n'a pas mis longtemps. Ça dépend toujours de la manière dont tournent les choses.

[As-tu le droit de pratiquer cette activité ?] On a le droit à la campagne, mais normalement on n'a le droit que pour sa propre consommation ou pour un voisin. On n'a pas le droit d'en faire du commerce. Mais pour ceux-là, on a le droit, ils ne sont pas bouclés ni rien. Ils sont tous déclarés consommation familiale.

[As-tu déjà eu des ennuis ?] Moi, je n'en ai jamais eus. D'autres, beaucoup d'autres en ont eus. Moi, j'ai eu plus de chance que les autres : je n'en ai jamais eus. Je m'en fiche! Celui qui n'est pas content n'a qu'à venir me remplacer. Moi, je voudrais laisser un autre le faire, apprendre à quelqu'un d'autre à faire le travail. [Trouves-tu des personnes pour transmettre le métier ?] Oh, on aura du mal à trouver! Quelqu'un me disait hier dans une ferme: " Quand

18. Mot-à-mot : la petite peau.

19. Comparaison aux autres cochons tués par le boucher dans cette ferme les semaines précédentes.

20. Mot-à-mot : l'intestin aveugle. Et oui, trouvé ! 
tu ne viendras plus, comment fera-t-on? »C'est un métier difficile et il va s'arrêter comme les autres aussi. Il faut savoir les tuer. Il ne faut pas avoir peur, quoi. Moi, j'ai commencé ce métier.., ce mois-ci, ça fera quarante-neuf ans que je fais ce métier.

[Te souviens-tu de la première fois ?] Oh oui ! Comme si c'était hier ! Il fallait avoir un bon caractère, avoir du cran surtout. Mais bon, moi, je suis de la campagne. Et pourtant, quand on tuait le cochon autrefois à la maison, je ne restais pas pour l'abattage, je partais. Mais depuis... C'est comme une infirmière ou un médecin : ces gens-là entaillent les gens, pareil. Ça, j'aurais du mal à le supporter, tu vois. Moi, je n'ai qu'un cochon entre les mains. Ceux qui ont la vie d'un homme entre les mains, c'est pire. Mais bon, quelqu'un doit bien le faire. Travailler dans les abattoirs, ce n'est pas non plus un travail des plus agréables. Quand on voit le marchand, comme ça, qui expose sa boucherie et sa charcuterie, ou des choses comme ça, ça présente bien, mais on n'imagine pas quel travail il y a eu auparavant.

\subsection{La découpe du cochon}

« Du boudin. On va faire du boudin. On fait du boudin avec du sang, du lard et des oignons. Pour deux kilos de sang de cochon : deux kilos d'oignons, un kilo et demi de gras et ensuite environ trois litres de sang, quatre litres de sang. C'est quand on tue le cochon qu'on récupère le sang. Il faut laisser l'oignon et le gras cuire d'abord, qu'ils rôtissent un peu.

On commence toujours à découper le cochon de la même manière, on coupe d'abord quatre ou cinq morceaux, pour faire de grandes parts, avant de les découper en petits morceaux. J'ai entendu dire « disober» aussi [pour « découper le cochon»], mais je dis toujours « dispenn ». Moi, j'ai toujours entendu « dispenn ar penoc'h».

Je retire le filet. Autrefois, quand on découpait le cochon, c'était toujours ce morceau qu'on mangeait le jour de la découpe. Ça, c'était toujours la fête autrefois, la tradition. La cuisinière préparait toujours ce morceau au déjeuner. Et n'importe où se rendait le boucher, autrefois c'était comme cela. La tradition consistait à manger ce morceau, en compagnie du boucher.

Mais les choses ont changé maintenant. La découpe ne se fait plus de la même manière. Autrefois quand on découpait le cochon, on mettait toute la viande - il n'y avait pas de congélateur - on mettait toute la viande dans un pot. Et on séparait la viande maigre de la viande grasse, parce qu'on mettait la viande grasse au fond du pot. Sinon, on faisait aussi des panneaux de côtes de poitrines ${ }^{21}$ qu'on suspendait. Mais les cochons étaient beaucoup plus lourds autrefois qu'aujourd'hui, beaucoup plus gras, parce que, plus les cochons étaient gras, plus on disait que la maison était riche. Quand on voyaient le lard suspendu, on se disait « Tiens, ça c'est une bonne maison! »

Ça, c'est la poitrine. C'est-à-dire les côtes de poitrine. Il faut la détacher de l'épaule.

21. Mot-à-mot : « côtelettes de lard salé ». Cela s'est fait jusque dans les années 1970 : de grands panneaux de lard blanc, salé et poivré sur un lit de paille et une toile de jute, d'environ $50 \mathrm{~cm}$ x $100 \mathrm{~cm}$ et de $10 \mathrm{~cm}$ d'épaisseur de gras. 
On ne faisait pas de côtelettes comme aujourd'hui, on faisait des morceaux de dos ${ }^{22}$. Pour faire des morceaux de dos, on coupait les côtes au raz de la colonne vertébrale de la bête, des deux

côtés on laissait du gras jusqu'au milieu du dos. Et on mettait ça toujours sur le dessus du pot pour pouvoir les manger en premier. Souvent, la tradition de partage avec les voisins consistait à donner un morceau de dos au voisin ou à la famille, ou quelque chose comme ça, pour leur faire plaisir. Car c'était ça le meilleur morceau. Et ensuite, quand les voisins découpaient à leur tour, il y avait souvent une compensation, on redonnait un morceau. C'est comme ça qu'on avait l'occasion de manger de la viande frâiche plus longtemps. Puisqu'il n'y avait rien pour la conserver.

Chaque couteau, normalement, a sa fonction. Certains couteaux servent à désosser, d'autres à dépecer la viande, et d'autres à la trancher pour faire des grands morceaux. Et la hache pour couper les os. Et la feuille pour couper les côtelettes. La feuille, c'est une petite hache. Sinon, pour les bêtes à cornes il faut le couperet, beaucoup plus grand. Aiguiser les couteaux, ça c'est un autre métier. Il faut savoir le faire. Si tu ne les aiguises pas bien, ce sera ensuite à tes dépends. Ça arrive parfois d'être touché. Ça fait parti du métier. Le pire coup que j'ai eu, c'est un coup de couteau dans la jambe. Alors que j'étais en train de saigner le cochon, comme je viens de faire là. Et il s'est passé que le cochon est allé... Dans les abattoirs, il y a des trous pour récupérer le sang, des trous dans le ciment, pour pouvoir mettre un seau dans ce trou rond, comme ça. Tu y mets donc le seau pour récupérer le sang. Et le cochon était allé dedans, comme ça, et moi, je tenais le pied du cochon comme je fais à celui-ci. J'avais le couteau à la main. Et le cochon n'était pas encore mort. Et quand j'ai voulu lui extraire le pied de là, avec un couteau comme celui-ci, le cochon a eu un geste brusque vers moi comme ça et pof, le couteau a glissé. Ça avait duré sept mois cette histoire. Mais je ne m'étais pas mis en arrêt. Maintenant, il y en a beaucoup qui s'arrêtent pour moins que ça.

Quand on est du métier - ceux qui sont du métier - nous reconnaissons un bon cochon, un cochon gras. À sa forme. Autrefois, quand on achetait des bêtes à la campagne, puisqu'on achetait des bêtes à la campagne, elles étaient achetées à l'amiable, on les achetait selon le classement. Il n'était pas nécessaire d'en savoir davantage puisque ensuite elles étaient amenées dans les abattoirs. Mais nous, autrefois, quand on les achetait, on les achetait avec nos yeux. On les achetait à un prix approximatif23. C'était à nous de savoir si c'était un bon ou non. Et quel poids il faisait à peu près. Je me souviens que, d'après la forme des bêtes, autrefois, à quelque chose près - oh oui à cinq kilos près - je savais quel poids de viande faisait la bête lors de l'abattage. Autrefois, lorsqu'il y avait des bovins, lorsque le marchand venait les acheter, il demandait très souvent au propriétaire de sortir les bêtes de la crèche pour en estimer le poids. Car le coup d'œil n'est pas le même selon les crèches : si la crèche à un toit haut, il a l'impression que la bête est beaucoup plus petite, et dans une petite crèche il a l'impression qu'elle est beaucoup plus lourde. La forme d'un bon cochon? On le voit bien à ses fesses, bien

22. Je ne connais pas le terme français pouvant correspondre à ce morceau fait à partir d'une coupe à la scie le long de la colonne vertébrale, en conservant la moitié de la noix et le gras du dos.

23. Mot-à-mot : « au prix de travers ». 
tournées, et à sa colonne vertébrale. Surtout à sa colonne vertébrale. Normalement pour avoir un bon cochon, il faut avoir un cochon allongé. Et pour un cochon extra, le centre de son dos sera pour ainsi dire fendu, il y aura comme une rigole tout le long de son dos. Dans ce cas, il sera vraiment très bon. C'est pour cela que le coup d'œil a beaucoup d'importance. [La couleur de la viande ?] Oh oui, ensuite, oui. Mais bon, la viande, sauf dans le cas des cochons entiers maintenant les cochons sont tous castrés, sinon, la viande était beaucoup plus grasse - sinon, la viande de cochon est quasiment blanche.

[Des malformations ?] Ça, ça m'est souvent arrivé : des côtes cassées. Souvent, petits, leur mère leur a marché dessus. Et ils sont un peu déformés. Mais ce n'est que mieux, ils se portent eux-mêmes. Autrefois, il était aussi un peu question de la tuberculose. Je me souviens, quand on travaillait pour tuer les bêtes, quand les poumons étaient collés aux côtes - et dans les abattoirs, c'est toujours comme ça - c'était déclaré « suspect». Nous, nous savions aussi bien que les vétérinaires si la viande était consommable ou pas. Nous avons vu un peu de tout dans notre métier. Les bêtes sont toutes à peu près formées de la même manière, à quelque chose près. Par contre, les rognons, ça je l'ai souvent vu : c'est pour les cochons comme pour les hommes, il n'y en avait parfois qu'un, ou bien un autre desséché. Ils sont peut-être nés comme ça. Et le deuxième était alors deux fois plus gros. Sans doute parce qu'il avait deux fois plus de travail. On les coupe en plus pour faire la charcuterie, les saucisses, ou encore l'andouille, l'andouille de viande. On fait moins d'andouille parce qu'il y a moins de cheminées pour les fumer.

[Des bêtes diformes ?] Non. Je n'ai pas vu ça. Avant de voir des choses comme ça, souvent elles ne vivent pas vieilles, elles meurent sans doute avant. J'en ai vues des accidentées. Autrefois, comme l'abattage à la campagne était autorisé, régulièrement, surtout à cette époque de l'année, j'en avais plein à abattre. Lorsqu'on sortait les génisses, elles allaient dans les chemins creux et se cassaient les jambes. Parce qu'on élevait les bêtes dans des crèches sombres autrefois, parce qu'il n'y avait pas d'ouverture. Parce que autrefois il fallait boucher les fenêtres pour que les bêtes aient chaud. Maintenant, c'est l'inverse, maintenant il faut ventiler. À l'époque quand on les sortait, elles ne voyait pas clair. Donc à cette époque de l'année, il y avait de nombreuses vaches... Quand les vaches vèlaient, elles faisaient parfois des hémorragies internes. Combien de fois n'ai-je pas été appelé en pleine nuit pour tuer une vache! Je n'ai pas dormi tous les soirs dans mon lit! Quand je les tuais, j'en retirais la peau, elles n'étaient pas plus mauvaises à manger. Maintenant, il n'y a plus le droit de tuer ces sortes de bêtes, c'est l'écarissage qui vient. Mais autrefois, les gens..., car le vétérinaire m'appelait, parce que, quand il arrivait - souvent on demandait au vétérinaire de venir voir la bête - s'il n'y avait rien à faire, c'est lui qui nous sifflait, pour soulager le propriétaire de la bête, parce que autrefois les gens n'étaient pas riches, et perdre une bête, ça coûtait cher. Les choses ont changé. C'est un sacré métier! Et il n'y guère de concurrence ! Il n'y a plus personne!

Le gras qui se trouve sur la colonne vertébrale sert à faire le pâté. Souvent, on met toute la viande grasse dans le pâté. On met tout le gras en morceaux parce que, quand on fait du pâté de campagne, on met environ deux kilos de viande grasse pour un kilo de foie et de cœur.

Autrefois, quand ont tuait un cochon, il n'y avait pas de perte, parce que - moi, je me souviens de voir mes parents faire eux-mêmes car le boucher venait (et il y avait un boucher dans chaque quartier) - il ne faisait que découper le cochon et préparer le pot de viande. C'était ensuite à chacun de faire la charcuterie lui-même. J'ai vu mettre dans le pâté jusqu'à la 
couenne mal cuite ou à moitié cuite. Maintenant, on ne la met plus : les gens sont difficiles ! On ne fait plus beaucoup de saindoux. J'ai en fait il y a peu car j'avais trouvé un beau morceau. Comme ça, pour mettre sur la plaque pour faire des crêpes. Ou quand on fait des pommes-deterre nouvelles au chaudron. La panne de gras fumé, lui, est suspendu. Quand on suspendait ça autrefois, on appelait ça «broneg ». C'était entouré d'un filet et mis à sécher, et on en coupait des morceaux au fur et à mesure. Et le « lard doux », c'est du lard non salé.

Le jarret : quand c'est un peu salé, alors on ne recule pas devant la gamelle !24 Il y a des pays qui font du jambon sec, mais ici, on ne peut pas faire ça. Le temps est trop humide. Ils pourrisent et ne sèchent pas. Les rôtis, rôtis de porc. Je vais faire les côtelettes aussi. Et parfois, on appelle ça l'échine, le cou du cochon. J'extrais les côtes pour découper des tranches de lard ensuite.

Maintenant, je vais faire le pâté de campagne. [Il énumère les morceaux qui se trouvent dans une bassine.] Voilà la cervelle. Quelqu'un veut avoir la cervelle? Personne ? Vous êtes assez intelligents? Voilà le péritoine qu'on pose sur le pâté. Il est attaché à l'estomac. Et on le met sur le dessus du pâté ensuite. L'estomac, on l'appelle la panse. Le cœur. Les poumons. Autrefois, on les mettait également dans le pâté. Autrefois, on ne perdait rien. Les poumons doivent se dire « ar skevent » en breton. Le foie. [On met le foie dans le pâté ?] Avec quoi veuxtu faire du pâté ? Si tu n'as pas de foie, tu n'as pas de pâté ! C'est du pâté de foie. Il y a le pâté de tête et le pâté de cochon, ou le pâté de l'autre bout ${ }^{25}$, si tu veux. Le pâté est prêt. Il ne reste plus qu'à le hacher.

[Le hachoir mécanique.] Je me suis servi de ça pour hacher au début de ma carrière. Ça n'avance pas! Et il fallait de la force. Et avoir une table solide parce qu'il fallait attacher la machine à la table. Une fois, dans une ferme, la table était attachée à une espèce de cloison, et à chaque tour de manivelle, elle se détachait un peu de la table: pourrie, quoi! Mais dans presque toutes les maison, il y a un hachoir comme ça : un hachoir mécanique dans toutes les fermes.

[Les boyaux.] Autrefois et même aujourd'hui, c'est avec l'intestin grêle ${ }^{26}$ que l'on fait les saucisses. Et autrefois, c'était avec le gros intestin ${ }^{27}$ que l'on faisait l'andouille de viande que l'on suspendait. Mais aujourd'hui, on ne s'amuse plus à les nettoyer. Si, je connais deux personnes qui font encore des andouilles de boyaux. Mais bon, je n'en fais plus beaucoup. Aujourd'hui, les grandes andouilles sont faites dans des boyaux de vaches et les boyaux à saucisse sont des boyaux de cochon. Tout à l'heure, c'est à voir, je ferai peut-être des saucisses fines, des chipolatas, et elles sont faites dans des boyaux de moutons. La viande est la même, mais les boyaux sont différents. De nos jours, les jeunes préfèrent les saucisses fines. Pourtant, les grosses saucisses ont bien meilleur goût. [Tu laves les boyaux ?] Je n'essaie même plus de

24. Mot-à-mot : «on n'est pas des fainéants à table! »

25. En breton, «penn » signifie « tête » et « extrémité, bout », d'où le jeu de mot du boucher : il y a le pâté de tête qui est l'une des extrémités du corps de la bête et le pâté de l'autre extrémité.

26. Mot-à-mot : « intestins fins».

27. Mot-à-mot : « grands intestins » et « intestins épais». 
le faire parce que ça prend trop de temps. C'est pour ça que les boyaux coûtent cher et qu'on les fait industriellement. Je les lavais à la main autrefois.

Il y a le gros intestin, mais ensuite tu as un boyau qui est comme un sac au bout, qu'on qualifie de poche : «la poche d'appendice». Autrefois, je me demande si on n'y faisait pas le saindoux fumé. On faisait ça avec du saindoux qu'on empaquetait et qu'on mettait à fumer dans la cheminée, et qu'on laissait en suspend comme ça. Autrefois, les gens en coupaient des morceaux pour mettre dans la soupe. On aurait du mal à trouver des amateurs de cette choselà aujourd'hui. Le coecum, c'est un intestin aveugle, oui. De cette taille-là environ [montrant une longueur de 20 centimètres environ avec sa main]. Il forme une poche dans laquelle va la nourriture, comme un sac vide. Et ensuite, elle doit faire demi-tour pour sortir par ce trou. Et nous, nous sommes comme ça aussi. Je n’ai pas été voir et je ne voudrais pas aller voir, d'ailleurs!

[La tête est cuite dans un grand pot.] C'est drôlement chaud ! Je n'y vois rien à l'intérieur. C'est la pause de 10h. [Une pause, un verre de vin rouge pour attendre que la tête refroidisse un peu.] On a repris un peu de force, alors reprenons maintenant. [N'as-tu pas faim ?] Oh non! Moi, je mange bien le matin. Et puis, des choses comme ça, ça n'ouvre pas l'appétit.

[Le pâté de campagne.] Allez, du sel et du poivre. Tu sales ça comme tu sales la soupe, approximativement. Je ne goûte pas. Je goûterai le pâté de tête tout à l'heure.

[Qu'est-ce qui est bon à manger dans la tête ?] Tout sauf les os. Les joues, le groin et la langue. Pas les yeux, sinon le cochon continuera à te regarder ! Voilà l'œil. Quand c'est bien cuit, ce n'est pas dur à désosser. Il y a une manière de sortir les yeux, un sens. Il lui manque des dents! Le hachoir n'aime pas les os. Il y a du cartilage dans les oreilles.

[Le boudin.] Qui veut le goûter ? Ce n'est jamais que du vin épais ! Ce n'est pas mauvais! Si l'eau est bien chaude, il faut dix à quinze minutes de cuisson. Si l'eau est très chaude. Moi, je le cuits dans le bouillon de cuisson de la tête. Le boudin, il faut le surveiller un peu. Il ne faut pas que ça commence à bouillir sinon c'est mauvais signe : ils éclatent alors tous, il n'en restera pas un entier.

[Comment as-tu appris ton métier ?] J'ai appris mon métier sur le tas, en apprentissage et des cours par correspondance, la technologie c'était tout par correspondance. J'étais à l'école Gambetta de M., une journée tous les quinze jours : Oh! Ça ne fait pas beaucoup! À l'époque, on faisait l'apprentissage en trois ans et au bout de deux ans, j'ai passé mon CAP de boucherie, et comme il y avait trois ans d'apprentissage à faire, l'année suivante j'ai fait la charcuterie. Le niveau scolaire n'était pas des plus élevés. De nos jours, ceux qui sortent de l'école, pour le CFA à B., passent beaucoup plus de temps à l'école qu'ils ne travaillent dans le métier. Nous, au bout de trois ans, nous étions aptes à faire n'importe quoi. Alors que ceux qui sortent des collèges, ils ont encore le métier à apprendre. On apprend des choses à l'école, mais entre l'école et la réalité du métier ensuite... sur le terrain, ce n'est pas pareil.

J'ai beaucoup fait de vaches. J'ai tué des chevaux également. J'ai tué des chevaux pour les assurances. Car autrefois, les chevaux, à la campagne, étaient assurés, et il y avait des groupements, tous les voisins, le secteur d'ici faisait partie de X. Lorsqu'un cheval mourrait, mettons qu'il y avait cinquante propriétaires de chevaux, et quand il manquait un cheval quelque part, on évaluait le cheval une fois l'an. Ça se faisait toujours le lundi de Pâques. Lors du rassemblement, dans un carrefour, on estimait chaque cheval. Comme ça, quand un cheval mourrait dans l'année... Et ensuite, chacun participait au remboursement du cheval en proportion du nombre de chevaux qu'il avait. S'il en avait trois, mettons sur cent chevaux, 
on divisait. Chacun donnait une petite somme d'argent. Un peu d'argent, pour remplacer le cheval. Parce que les chevaux avaient de la valeur autrefois. Par la suite, il y a eu des assurances, comme les assurances qu'on connaît de nos jours. Mais bon, maintenant il n'y a plus de chevaux nulle part. J'ai donc tué des chevaux parce que, à l'époque, il y avait ce qu'on appelle la «chair de poisson ». Cela vient de la paralysie. Ça arrivait souvent aux bêtes à cornes, je l'ai vu lorsqu'elles étaient accidentées. Les muscles, lorsqu'ils sont atrophiés ou déchirés, formaient comme une boule de graisse. Ça arrivait aussi aux chevaux lorsqu'ils étaient usés car les chevaux travaillaient beaucoup autrefois, et on appelait ça de la chair de poisson parce que la viande devenait blanche. Et pour être remboursé par l'assurance, il fallait qu'ils soient morts. Les gars des assurances faisaient appel à moi pour les tuer. Je n'étais pas payé, je faisais ça bénévolement.

[Tout en tournant les boyaux pour faire les saucisses.] Le poussoir : une presse. On ne mettra pas tout dedans en une fois, il faudra faire deux tournées. Ces boyaux sont des intestins de cochon. Là, j'ai des boyaux de vaches pour faire l'andouille. Ils sont beaucoup plus épais, leur diamètre est beaucoup plus grand. Allez, super! Comment ça se passe le partage des saucisses? Ça, je l'ai fait de nombreuses fois: des tours et des tours, des tonnes! Quand je sais à peu près de quel poids je dispose, je sais à peu près combien de saucisses je vais faire avant de me lancer. Normalement, avec ce que j'ai là, je devrais en faire environ cent quarante. Les cochons, ça ne change pas. Je dis ça tout le temps : les gens ont changé mais la nature n'a pas changé.

\section{Pour clore le propos : ce cul-de-sac intestinal}

En cherchant l'équivalent breton de l'heste itsu basque, j'ai non seulement trouvé un autre intestin aveugle sous l'appelation de bouellenn dall2 ${ }^{28}$, mais un grand nombre d'autres dénominations de cette partie de l'intestin motivées par la cécité : l'allemand Blinddarm, l'anglais blind gut ou typhlitis, l'espagnol el intestino ciego, l'italien intestino cieco, le nom coecum ${ }^{29}$ luimême qui vient du cœcum intestinum latin, signifiant « aveugle, sombre, sans ouverture », certaines lésions du cœcum qui sont qualifiées à partir du grec «typhlo- » signifiant « aveugle », etc. Un grand tour des dénominations de cet intestin dans les langues d'Europe ou d'ailleurs serait fort intéressant pour en saisir l'étendue des diverses motivations. En attendant, ce petit tour dans le monde de la boucherie-charcuterie aura permis également d'apprendre - en plus des pratiques et du vocabulaire de ce domaine en breton - que le cochon n'est pas exposé à l'appendicite car son « intestin aveugle », contrairement à celui de l'homme, ne se prolonge pas par un appendice.

28. En breton, ce qualificatif est d'ailleurs assez fréquemment employé dans le sens de cul-desac comme dans park dall (champ bouché), hent dall (cul-de-sac) ou encore kontell dall (couteau émoussé).

29. /sekom/ 